Classification

Physics Abstracts

$72.70-72.80-02.90$

\title{
Determination of PHEMT's Microwave Noise Parameters Only by Means of the Small-Signal Equivalent Circuit and Experimen- tal Comparisons
}

\author{
D. Gasquet $\left({ }^{1}\right)$, F. Barberousse $\left({ }^{1}\right)$, M. de Murcia $\left({ }^{1}\right)$, W. de Raedt $\left({ }^{2}\right)$ \\ and C. Claeys $\left({ }^{2}\right)$ \\ ( $\left.{ }^{1}\right)$ Centre d'Electronique de Montpellier, UM2, 34095 Montpellier cedex 05, France \\ $\left({ }^{2}\right)$ IMEC, Kapeldreef 75, B-3001 Leuven, Belgium \\ (Received 8 July 1994, accepted 3 November 1994)
}

\begin{abstract}
Résumé. - Le but de cet article est de donner une méthode analytique de modélisation des paramètres de bruit de transistors à effet de champ et plus particulièrement de HEMT pseudomorphiques. L'originalité de cette méthode tient dans le fait qu'elle ne comprend pas de paramètres ajustables et qu'elle ne nécessite que la connaissance des éléments du schéma équivalent petit signal. Elle prend en compte toutes les sources de bruit thermique associées aux résistances, le bruit du canal étant décrit par un générateur de courant de bruit de densité spectrale $4 k T_{0}\left(g_{\mathrm{m}}+g_{\mathrm{ds}}\right)$. Les résultats théoriques sont comparés à nos mesures mais aussi a une autre technique de mesure faisant intervenir une part de modélisation (Dambrine [7]). Nous montrons que l'accord est excellent pour les quatre paramètres de bruit aussi bien en fonction de la fréquence qu'en fonction de la polarisation.
\end{abstract}

\begin{abstract}
The purpose of this paper is to develop an analytical method in order to model the noise parameters of pseudomorphic HEMT's. The principal interest of this method is that it does not need adjustable parameters and it only needs the knowledge of the components of the small signal equivalent circuit. It takes into account all the thermal noise sources associated to the resistors and the noise of the channel is described by a noise current source with a spectral density $4 k T_{0}\left(g_{\mathrm{m}}+g_{\mathrm{ds}}\right)$. The theoretical results are compared to our measurements but also to other measurement technique involving a part of modelization (Dambrine [7]). We show that the agreement is excellent for the four noise parameters as well versus the frequency as versus the bias.
\end{abstract}

\section{Introduction}

Many papers have been written on noise modeling GaAs FET's [1-7]. Unfortunately, in order to predict the noise behavior of a device, all these models either need the knowledge of noise measurements results [7] and/or the use of two or three adjustable parameters [1-6] in addition to the knowledge of the small signal equivalent circuit. We do not speak here about the 
physical microscopic models taking into account interactions and transport phenomena but about electrical models only. Our model is only based on analytical electrical calculations on condition that we know exactly the component values of the equivalent circuit (Fig. 1). So we will briefly explain here the method used to determine this equivalent circuit. Then the theoretical noise calculations will be compared to noise measurements performed on pseudomorphic HEMT's processed by IMEC. Noise measurement has been performed in the range 8 $\mathrm{GHz}-18 \mathrm{GHz}$.

\section{HEMT structure}

The layer sequence for PHEMT devices has been grown by using molecular beam epitaxy (MBE). A $1 \mu \mathrm{m}$ thick undoped GaAs buffer is grown on a semi-insulating GaAs substrate followed by the $13 \mathrm{~nm}$-thick undoped InGaAs (In\% $=20 \%$ ) active layer. The $30 \mathrm{~nm}$-thick GaAlAs $(\mathrm{Al} \%=22 \%)$ supply layer is separated from the active layer by a $5 \mathrm{~nm}$-thick undoped GaAlAs $\left(5 \times 10^{14} \mathrm{~cm}^{-3}\right)$ spacer in order to provide a high quality interface. To reduce parasitic resistances the $40 \mathrm{~nm}$ cap layer is highly doped. The carrier concentration in the channel is $1.6 \times 10^{12} \mathrm{~cm}^{-2}$ and the mobility at $300 \mathrm{~K}$ is $5800 \mathrm{~cm}^{2} / \mathrm{Vs}$.

The devices are processed by using standard technology. The gate pattern is defined by e-beam lithography resulting in a gate length of $0.2 \mu \mathrm{m}$. The surface is passivated by using SiN. Multi-gate-finger structure 4 fingers with a total length of $150 \mu \mathrm{m}$ has been used and the source pads are interconnected by air bridges.

The outer pad configuration has been designed for on-wafer testing with G-S-G probe tips. This structure exhibits high gain values and very low noise figure typically $g_{\mathrm{m}}=800 \mathrm{mS} / \mathrm{mm}$ and $\mathrm{NF}=0.48 \mathrm{~dB}$ at $8 \mathrm{GHz}$.

\section{Extraction of the equivalent circuit}

As we said in the introduction, in order to determine the noise parameters, it is necessary to obtain with a good accuracy the component values of the equivalent circuit. This equivalent circuit (Fig. 1) can be divided into two parts, the intrinsic one and the extrinsic one. The extrinsic components including the access inductances and resistances and the pad capacitors are assumed to be bias independents. In the intrinsic part the transconductance $G_{m}$ is taken frequency dependent varying as $g_{\mathrm{m}} \mathrm{e}^{-\jmath \omega \tau}, \tau$ is the transit time under the gate. These values are determined using the method proposed by Cappy [8] and Dambrine [9]. This method includes

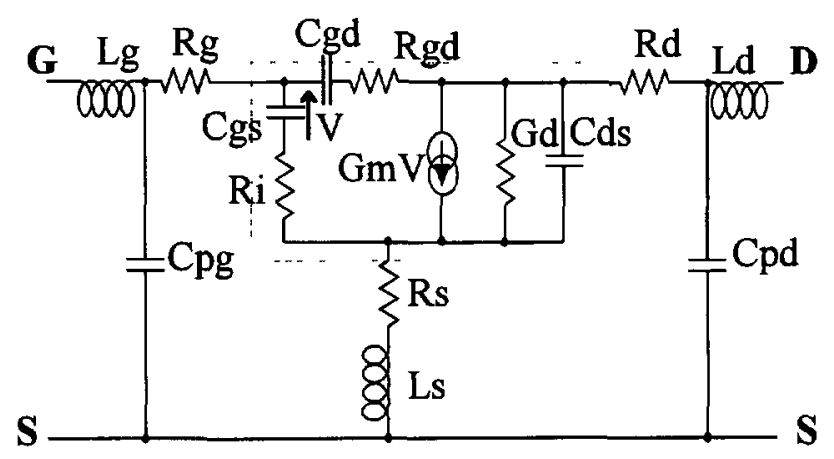

Fig. 1. - Equivalent circuit of the PHEMT. The dot rectangle define the intrinsic device. 
$\mathrm{C}_{\mathrm{ds}}(\mathrm{fF})$

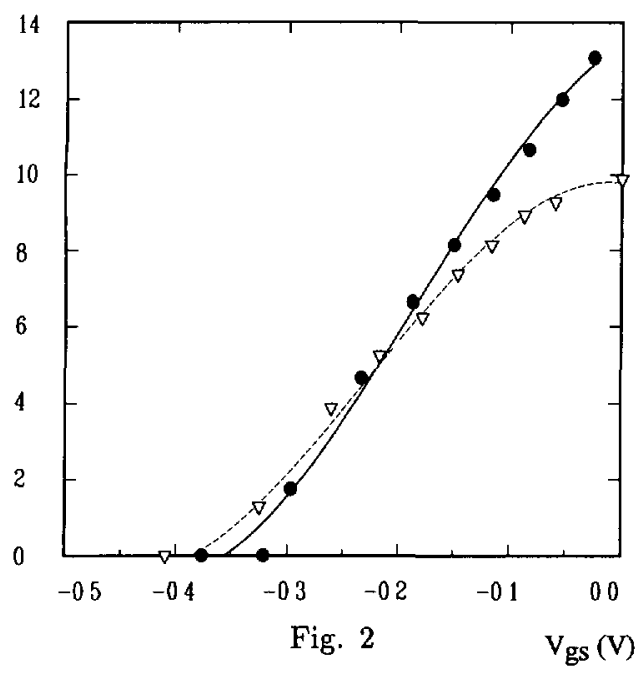

$\mathrm{C}_{\mathrm{gs}}(\mathrm{fF})$

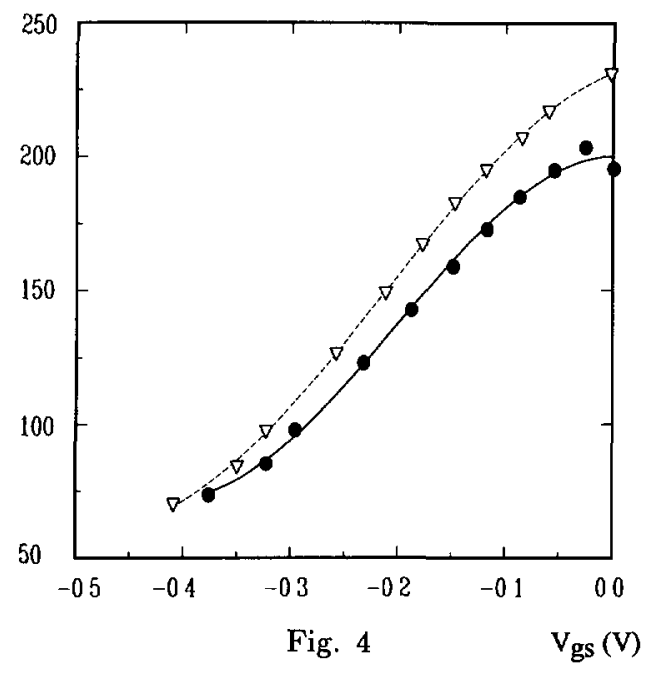

$\mathrm{C}_{\mathrm{gd}}(\mathbf{f} \mathrm{F})$

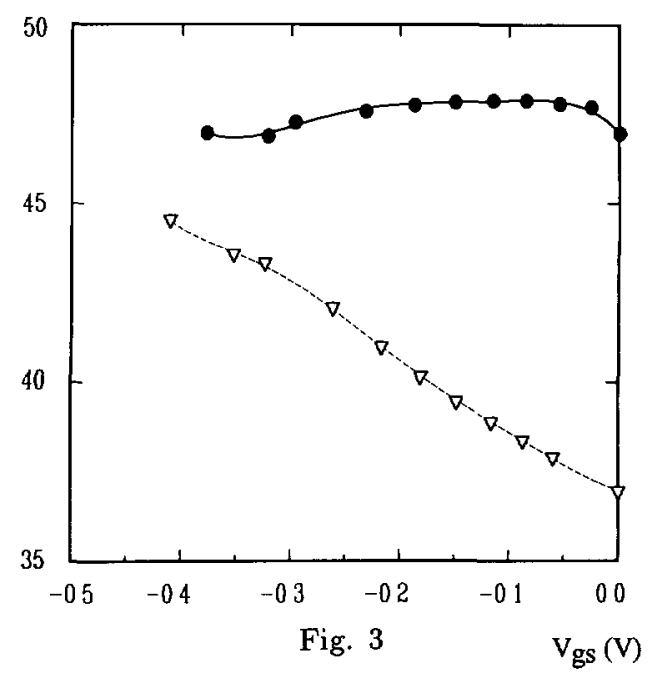

$\mathrm{G}_{\mathrm{m}}(\mathrm{mS})$

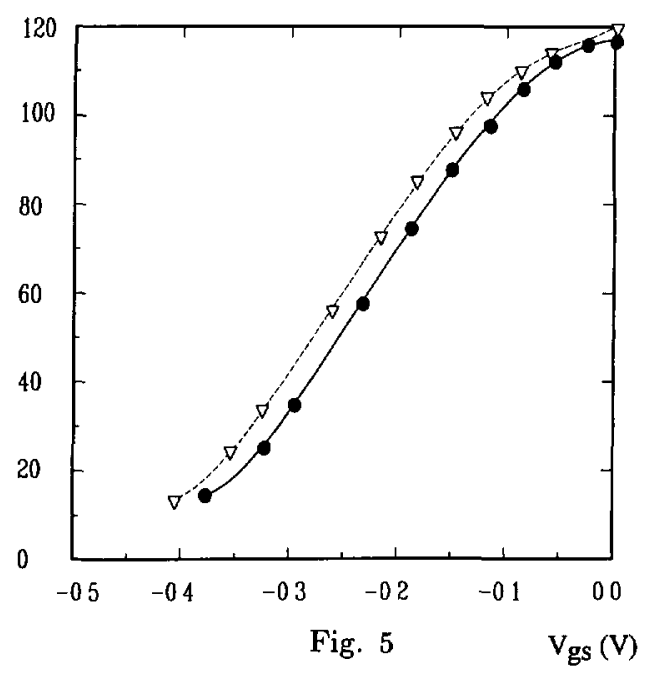

Fig. 2. - Drain source capacitance versus $V_{\mathrm{gs}} .(\bullet) V_{\mathrm{ds}}=1 \mathrm{~V},(\nabla) V_{\mathrm{ds}}=2 \mathrm{~V}$.

Fig. 3. - Gate drain capacitance versus $V_{\mathrm{gs}} \cdot(\bullet) V_{\mathrm{ds}}=1 \mathrm{~V},(\nabla) V_{\mathrm{ds}}=2 \mathrm{~V}$.

Fig. 4. - Gate source capacitance versus $V_{\mathrm{gs}} .(\bullet) V_{\mathrm{ds}}=1 \mathrm{~V},(\nabla) V_{\mathrm{ds}}=2 \mathrm{~V}$.

Fig. 5. - Transconductance versus $V_{\mathrm{gs}} .(\bullet) V_{\mathrm{ds}}=1 \mathrm{~V},(\nabla) V_{\mathrm{ds}}=2 \mathrm{~V}$.

two steps. In the first one we measure the scattering parameters of the device under special bias conditions [10] ( $V_{\mathrm{ds}}=0 \mathrm{~V}, V_{\mathrm{gs}}>0$, and $V_{\mathrm{ds}}=0$ under the pinchoff) in order to determine the parasitic elements. In the second step we measure the scattering parameters of the device under normal bias conditions and then by matrix transformation and subtractions of the parasitic 
$\mathrm{R}_{\mathrm{ds}}(\mathrm{K} \Omega)$

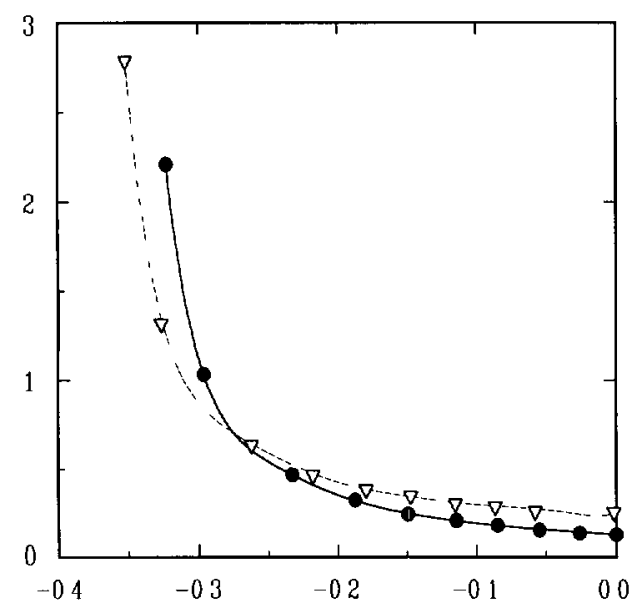

Fig. 6

$$
R_{i}(\Omega)
$$

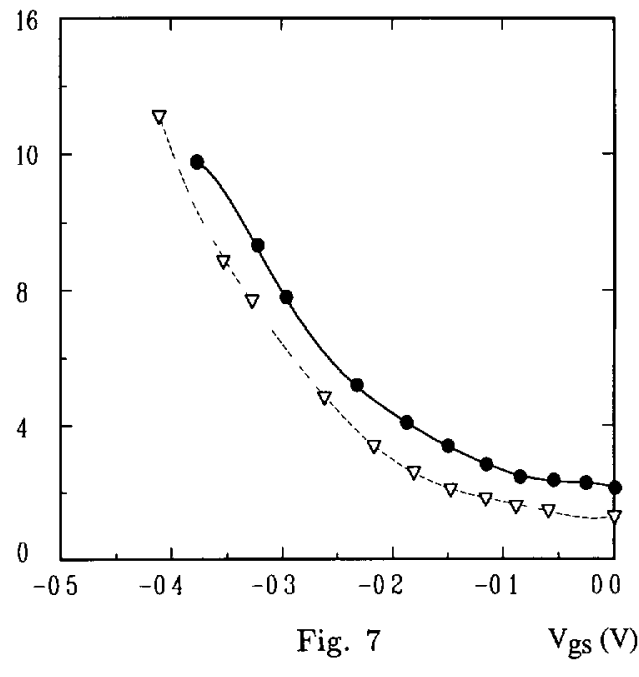

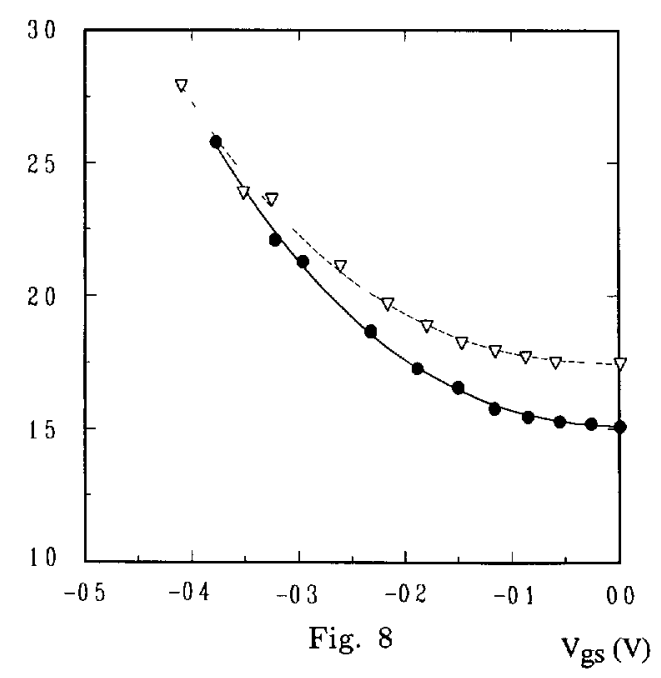

Fig. 6. - Drain source resistance versus $\left.V_{\mathrm{gs}} .(\bullet) V_{\mathrm{d} s}=1 \mathrm{~V}, \nabla\right) V_{\mathrm{ds}}=2 \mathrm{~V}$.

Fig. 7. - Input resistance versus $V_{\mathrm{gs}} .(\bullet) V_{\mathrm{ds}}=1 \mathrm{~V},(\nabla) V_{\mathrm{ds}}=2 \mathrm{~V}$.

Fig. 8. - Transit time under the gate versus $V_{\mathrm{gs}^{s}}(\bullet) V_{\mathrm{ds}}=1 \mathrm{~V},(\nabla) V_{\mathrm{ds}}=2 \mathrm{~V}$.

elements we obtain the admittance matrix of the intrinsic device. The values of the components are obtained by identification $[9,11]$. In Table I we give the extrinsic element values. We can notice the very low values found for the pad capacitances $\left(C_{\mathrm{pg}} \approx 0\right.$ and $\left.C_{\mathrm{pd}} \approx 30 \mathrm{fF}\right)$. The measurements were performed in range $6-18 \mathrm{GHz}$ directly on wafer. This fact explains the low inductance values found. Figures 2 to 8 give the behavior of $C_{\mathrm{ds}}, C_{\mathrm{gd}}, C_{\mathrm{gs}}, G_{\mathrm{m}}, R_{\mathrm{ds}}, R_{\mathrm{i}}$, and $\tau$ versus the bias conditions. From these results it is possible to evaluate the extrinsic transition 
Table I. - Values of the extrinsic elements. The value of $C_{\mathrm{pg}}$ is taken to be equal to 0 because the found value was $<0$.

\begin{tabular}{|c|c|c|c|c|c|c|c|}
\hline$L_{\mathrm{s}}(\mathrm{pH})$ & $L_{\mathrm{g}}(\mathrm{pH})$ & $L_{\mathrm{d}}(\mathrm{pH})$ & $C_{\mathrm{pg}}(\mathrm{fF})$ & $C_{\mathrm{pd}}(\mathrm{fF})$ & $R_{\mathrm{s}}(\Omega)$ & $R_{\mathrm{d}}(\Omega)$ & $R_{\mathrm{g}}(\Omega)$ \\
\hline 3.5 & 59.2 & 69.1 & 0.0 & 31.3 & 1.85 & 2.1 & 1.0 \\
\hline
\end{tabular}

frequency $f_{\mathrm{T}}[12]$ :

$$
f_{\mathrm{T}}=\frac{g_{\mathrm{m}}}{2 \pi \cdot\left\{\left[C_{\mathrm{gs}}+C_{\mathrm{gd}}\right]\left[1+\frac{R_{\mathrm{s}}+R_{\mathrm{d}}}{R_{\mathrm{da}}}\right]+C_{\mathrm{gd}} g_{\mathrm{ni}}\left(R_{\mathrm{s}}+R_{\mathrm{d}}\right)\right\}}
$$

This frequency is as high as $72 \mathrm{GHz}$.

\section{Noise calculations}

In order to perform noise calculations we have associated a noise voltage generator at each resistive element of the equivalent circuit. The spectral densities of these noise generators have been taken to be equal to $4 k R T_{0}$ where $R$ is the resistance value and $T_{0}$ is the room temperature. In addition to this, according to Van der Ziel [13-15], the noise of the channel is represented by a current noise generator $\left.<i_{\mathrm{m}}^{2}\right\rangle=4 k T_{0} g_{\mathrm{m}} \Delta f P(x, y)$ associated to another one $\left\langle i_{\mathrm{ds}}^{2}\right\rangle=4 k T_{0} g_{\mathrm{ds}} \Delta f$ or $\left\langle e_{\mathrm{ds}}^{2}\right\rangle=4 k T_{0} R_{\mathrm{ds}} \Delta f$. We have taken the constant $P(x, y)$ equal to 1 . Then the total noise current generator in the drain is $\left\langle i_{\mathrm{d}}^{2}\right\rangle=4 k T_{0}\left(g_{\mathrm{ds}}+g_{\mathrm{m}}\right) \Delta f$. Now the equivalent circuit given on Figure $9 \mathrm{a}$ takes into account the
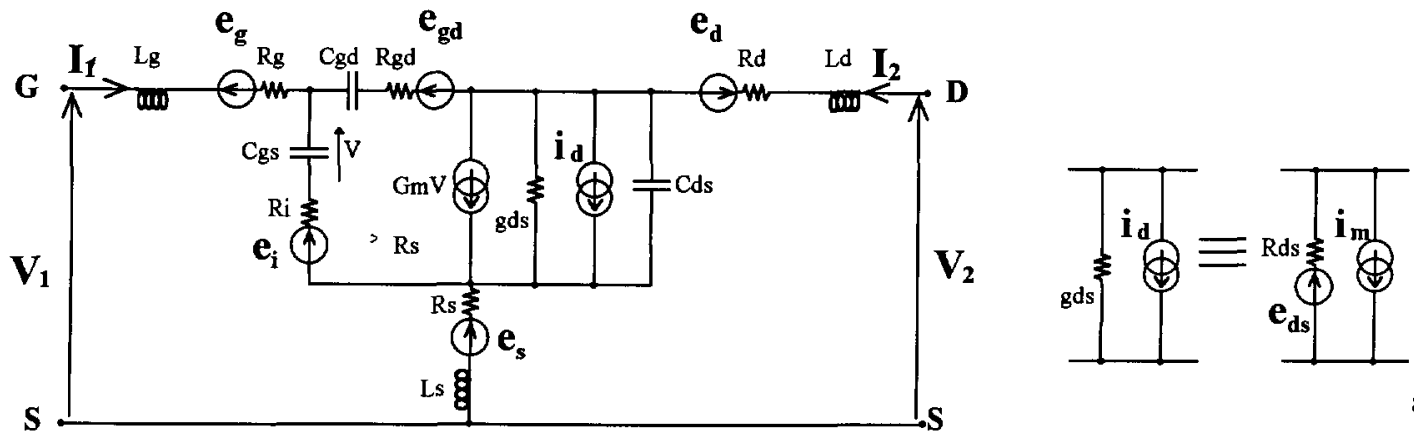

a)

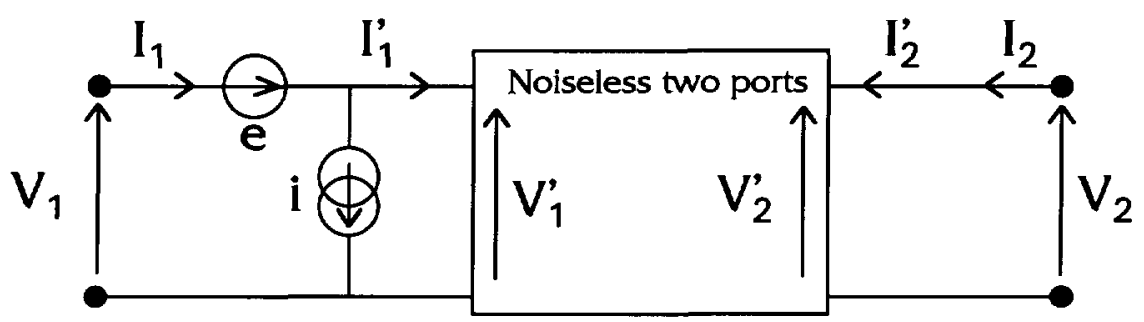

b)

Fig. 9. - a) Noisy equivalent circuit of the device. $e_{\mathrm{g}}, e_{\mathrm{gd}}, e_{\mathrm{d}}, e_{1}$ and $e d s$ are thermal noise voltage sources associated with resistances. $I_{\mathrm{m}}$ is a noise current source $4 k T g_{\mathrm{m}} \Delta f ; \mathrm{b}$ ) equivalent circuit of a noisy two port device. $e$ and $i$ are two correlated noise sources. 
noise sources which are uncorrelated, we only have neglected the pad capacitors. In order to determine the noise parameters of the device we have to transform this equivalent circuit in the one given on Figure $9 \mathrm{~b}$ therefrom, if we know $i$ and $e$, it will be easy to calculate the noise parameters as:

$$
\begin{aligned}
& F_{\min }=1+\frac{1}{2 k T \Delta f}\left\{\mathcal{R e}\left(\left\langle e i^{*}\right\rangle\right)+\sqrt{\left\langle e e^{*}\right\rangle\left\langle i i^{*}\right\rangle-\left[\mathcal{I m}\left(\left\langle e i^{*}\right\rangle\right)\right]^{2}}\right\} \\
& R_{\mathrm{n}}=\frac{\left\langle e e^{*}\right\rangle}{4 k T \Delta f} \\
& G_{\mathrm{opt}}=\sqrt{\frac{\left\langle i i^{*}\right\rangle}{\left\langle e e^{*}\right\rangle}-\left[\frac{\mathcal{I m}\left(\left\langle e i^{*}\right\rangle\right)}{\left\langle e e^{*}\right\rangle}\right]^{2}} \\
& B_{\text {opt }}=\frac{\mathcal{I m}_{\mathrm{m}}\left(\left\langle e i^{*}\right\rangle\right)}{\left\langle e e^{*}\right\rangle}
\end{aligned}
$$

The $Z$-matrix of the circuit (Fig. $9 \mathrm{~b}$ ) can be written as follow, if $Z_{\mathrm{ij}}$ are the $Z$ coefficients of the noiseless two ports:

$$
\begin{gathered}
V_{1}=Z_{11} I_{1}+Z_{12} I_{2}-Z_{11} i-e \\
V_{2}=Z_{21} I_{1}+Z_{22} I_{2}-Z_{21} i
\end{gathered}
$$

In the first step, considering the noise generators in the device circuit (Fig. 9a) as standard generators, we calculate the $Z$-matrix. This calculation is not very complicated but very tedious because we do not neglect any element except the pad capacitors. Then it is possible, by identification with (5) and (6), to obtain the expression of the generators $e$ and $i$. These expressions are quite complicated and are given here:

$i=\frac{i_{\mathrm{m}}\left(a_{1}+\mathrm{j} a_{2}\right)+e_{\mathrm{ds}}\left(b_{1}+\mathrm{j} b_{2}\right)+e_{\mathrm{s}}\left(c_{1}+\mathrm{j} c_{2}\right)+e_{\mathrm{d}}\left(d_{1}+\mathrm{j} d_{2}\right)+e_{\mathrm{i}}\left(e_{1}+\mathrm{j} e_{2}\right)+e_{\mathrm{gd}}\left(f_{1}+\mathrm{j} f_{2}\right)}{\alpha+\mathrm{j} \beta} \cdot C_{\mathrm{gs}} \omega$

$e=\frac{e_{\mathrm{g}}\left(g_{1}+\mathrm{j} g_{2}\right)+e_{\mathrm{B}}\left(h_{1}+\mathrm{j} h_{2}\right)+e_{1}\left(i_{1}+\mathrm{j} i_{2}\right)+e_{\mathrm{ds}}\left(j_{1}+\mathrm{j} j_{2}\right)+e_{\mathrm{gd}}\left(k_{1}+\mathrm{j} k_{2}\right)+e_{\mathrm{d}}\left(l_{1}+\mathrm{j} l_{2}\right)+i_{\mathrm{m}}\left(m_{1}+\mathrm{j} m_{2}\right.}{\alpha+\mathrm{j} \beta}$

The values of the variables $\alpha, \beta$ and $a_{\mathrm{i}}$ to $m_{\mathrm{i}}$ are given in Appendix A. These variables only depend on the circuit elements and frequency.

Now in the second step we calculate $\left\langle e e^{*}\right\rangle,\left\langle i i^{*}\right\rangle$ and $\left\langle e i^{*}\right\rangle$, where ${ }^{*}$ denotes the complex conjugate and \langle\rangle denotes the mean value. To perform these calculations we consider that the noise sources $e_{x}$ are uncorrelated thus the terms $\left\langle e_{x} e_{y}^{*}\right\rangle$ and $\left\langle e_{x} i_{y}^{*}\right\rangle$ vanish. The terms $\left\langle e_{x} e_{x}^{*}\right\rangle$ are equal to the thermal noise of the resistance $R_{x}\left(4 k R_{x} T_{0} \Delta f\right)$ and as we said earlier the term $\left\langle i_{\mathrm{m}} i_{\mathrm{m}}^{*}\right\rangle$ is equal to $4 k g_{\mathrm{m}} T_{0} \Delta f$. Under these assumptions we can express the noise parameters from equations (1), (2), (3) and (4) as follows:

$$
\begin{gathered}
F_{\min }=1+\frac{2 Q}{\alpha^{2}+\beta^{2}} \cdot C_{\mathrm{gs}} \omega+\frac{2 C_{\mathrm{gs}} \omega}{\alpha^{2}+\beta^{2}} \sqrt{S T-R^{2}} \\
R_{\mathrm{n}}=\frac{S}{\alpha^{2}+\beta^{2}}
\end{gathered}
$$




$$
\begin{aligned}
\left|Y_{\mathrm{opt}}\right|^{2} & =\frac{T}{S}\left(C_{\mathrm{gs}} \omega\right)^{2} \\
B_{\mathrm{opt}} & =-\frac{R}{S} C_{\mathrm{gs}} \omega
\end{aligned}
$$

The values of the variables $Q, R, S$ and $T$ are expressed in Appendix A. They only depend on the values of the elements of the small-signal equivalent circuit, so that under these assumptions it is possible to evaluate the noise performances of a given device only by means of the measurement of it equivalent circuit. The value of the optimal conductance $G_{\text {opt }}$ may be evaluated from the equations (11) and (12).

In order to calculate the noise parameters of the equivalent circuit it would be possible to use the correlation matrix approach [21]. This method avoids tedious calculations and leads directly to the results. Nevertheless it does not permit to obtain an analytical formulation of the results. Then, it is not easily possible to try to link noise parameters with technological parameters.

As we said in the introduction the literature proposes a lot of electrical noise modeling techniques. The most commonly used one is the Pospiezsalski's [1] approach. In this model, which uses only two noise temperatures $T_{\mathrm{g}}$ and $T_{\mathrm{d}}$, the noisy device is assumed to be represented by a noiseless device associated with an input noise voltage generator $\left\langle e_{\mathrm{g}}^{2}\right\rangle$ and an output noise current generator $\left\langle i_{\mathrm{d}}^{2}\right\rangle$. The expressions of these uncorreltated generators are:

$$
\begin{gathered}
\left\langle e_{\mathrm{g}}^{2}\right\rangle=4 k T_{\mathrm{g}} R_{\mathrm{i}} \Delta f \\
\left\langle i_{\mathrm{d}}^{2}\right\rangle=4 k T_{\mathrm{d}} R_{\mathrm{ds}} \Delta f
\end{gathered}
$$

From our model, using the transformation allowing us to obtain the Pospieszalski's representation, it is possible to express the temperatures $T_{\mathrm{g}}$ and $T_{\mathrm{d}}$ :

$$
T_{\mathrm{g}}=\frac{C_{\mathrm{gs}}^{2}}{\left(C_{\mathrm{gs}}+C_{\mathrm{gd}}\right)^{2}} T_{0}
$$

and,

$$
T_{\mathrm{d}} \approx\left(1+\frac{g_{\mathrm{m}}}{g_{\mathrm{ds}}}\right) T_{0}
$$

\section{Noise measurement}

The noise measurements have been carried out on wafer by means of a probe station "Alessi" coupled with a noise figure meter HP $8970 \mathrm{~B}$, a noise figure test set HP $8971 \mathrm{~B}$ and a synthesized $\mathrm{CW}$ generator HP $8673 \mathrm{G}$. At the input of the noise apparatus we have placed a low-noise amplifier and an isolator in order to diminish its noise figure and to keep its input impedance constant. The LNA has a noise factor lower than $4 \mathrm{~dB}$ and a gain higher than $25 \mathrm{~dB}$ in the range $1-18 \mathrm{GHz}$. In order to determine the noise parameters we use the multiple impedances method [16], [17]. These impedances are realized by means of an automatic mechanical tuner. This tuner is a home made one, concerning the magnitude of the reflection coefficient its repeatability is better than $2 \%$ and the uncertainty on its phase is in the worst case (at 18 $\mathrm{GHz}) 2$ degrees. In order to calibrate the tuner and deembed [18] the noise of the DUT we use a network analyzer HP 8720. This calibration step is very important and it must be performed with a great carefulness to avoid erroneous measurements. 
$\mathrm{F}_{50 \Omega}(\mathrm{dB})$

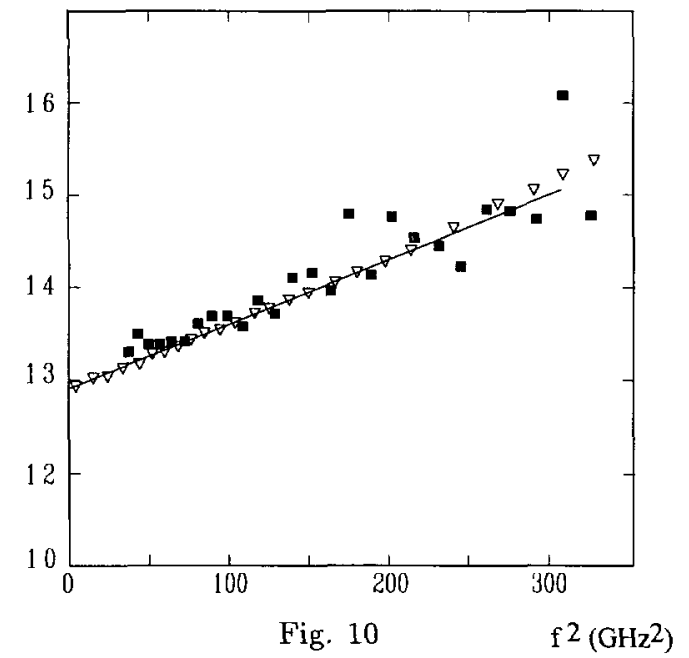

$\theta_{\text {opt (degree) }}$

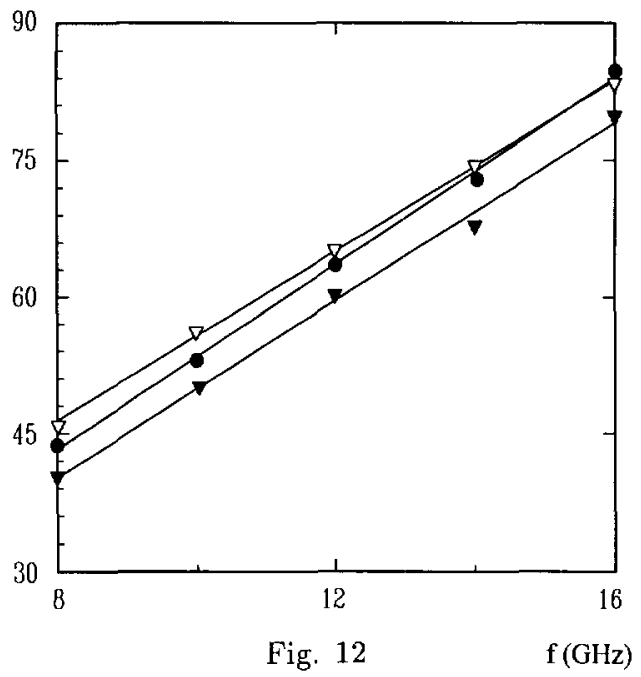

$\mathrm{F}_{\min }(\mathrm{dB})$

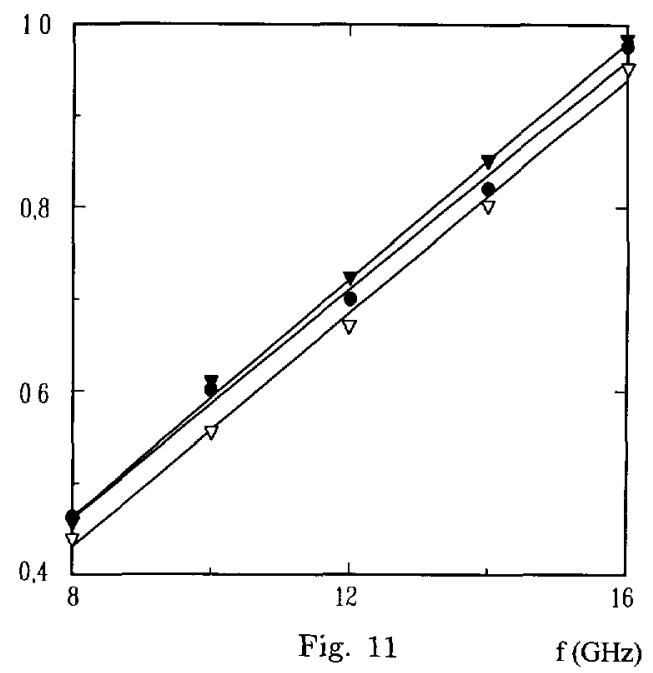

$\left|\Gamma_{\text {opt }}\right|$

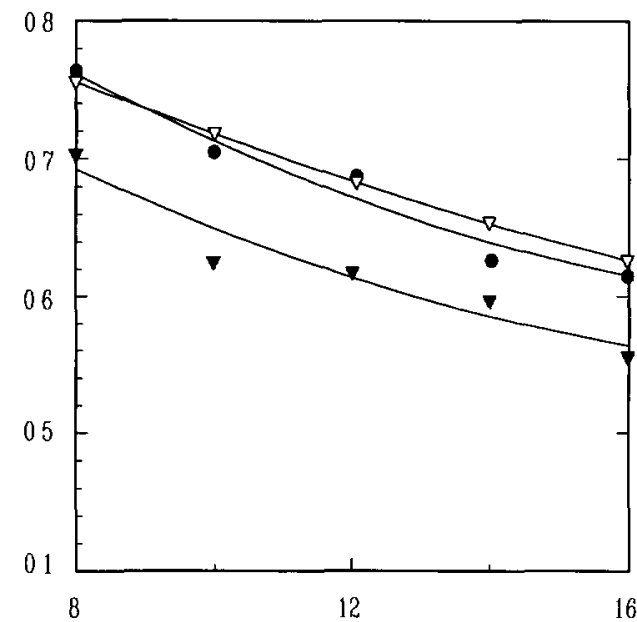

Fig. 13

$\mathrm{f}(\mathrm{GHz})$

Fig. 10. - Noise factor under $50 \Omega$. Condition versus $f^{2}, V_{\mathrm{ds}}=1 \mathrm{~V}, I_{\mathrm{d}}=26 \mathrm{~mA}$. (品) experimental results, $(\nabla)$ results of the calculation from equation (13) using $Y_{s}=20 \mathrm{mS}$.

Fig. 11. - Minimum noise figure versus frequency. $V_{\mathrm{ds}}=1 \mathrm{~V}, I_{\mathrm{d}}=9 \mathrm{~mA} .(\bullet)$ Experimental results, $(\nabla)$ Results of the calculation from equation $(9),(\boldsymbol{\nabla})$ Results obtained from Dambrine's method.

Fig. 12. - Phase of the optimum reflection coefficient versus frequency. $V_{\mathrm{ds}}=1 \mathrm{~V}, I_{\mathrm{d}}=9 \mathrm{~mA}$. $(\bullet)$ Experimental results, $(\nabla)$ Results of the calculation from equations (11) and (12), ( $\mathbf{\nabla})$ Results obtained from Dambrine's method.

Fig. 13. - Magnitude of the optimum reflection coefficient versus frequency. $V_{\mathrm{ds}}=1 \mathrm{~V}, I_{\mathrm{d}}=$ $9 \mathrm{~mA}$. (•) Experimental results, $(\nabla)$ Results of the calculation from equation (11) and 12, ( $)$ Results obtained from Dambrine's method. 

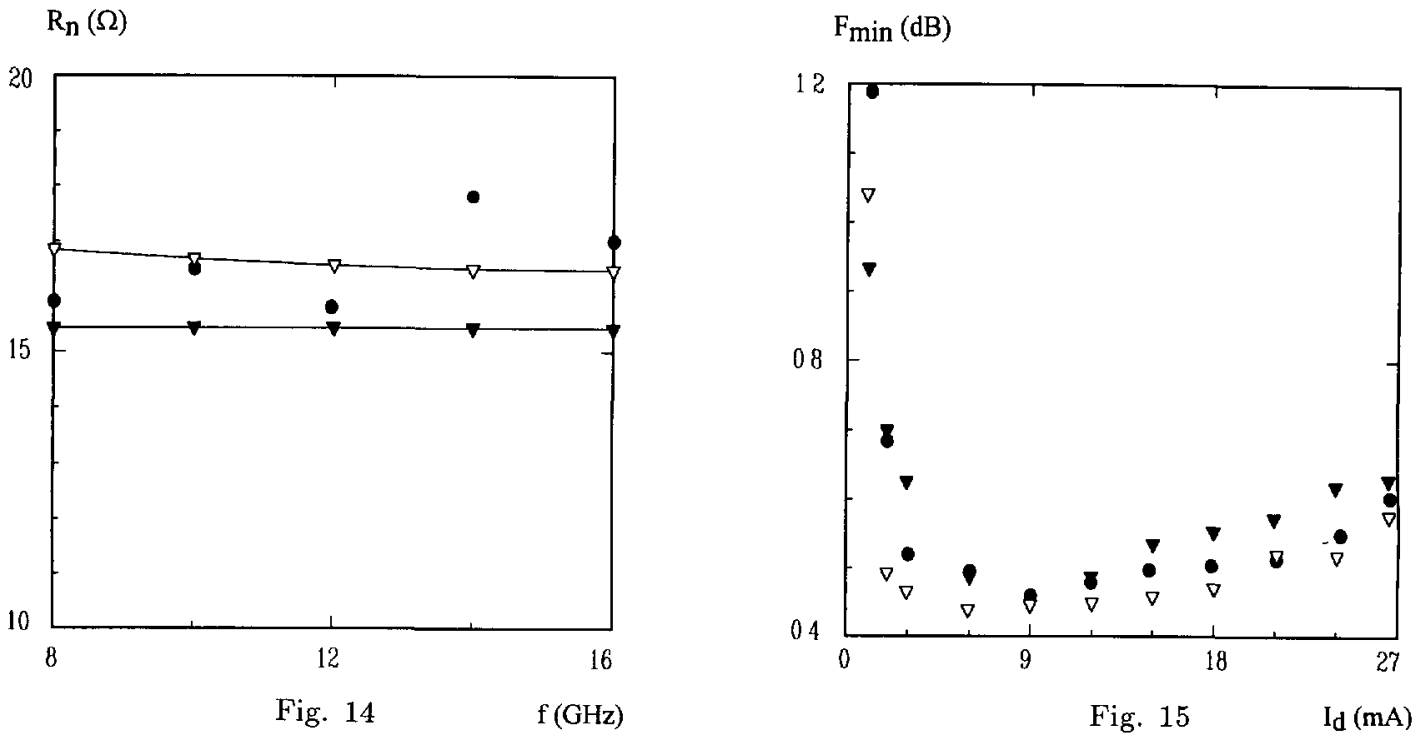

Fig. 14. - Equivalent noise resistance versus frequency. $V_{\mathrm{ds}}=1 \mathrm{~V}, I_{\mathrm{d}}=9 \mathrm{~mA}$. (•) Experimental results, $(\nabla)$ Results of the calculation from equation (10), ( $\nabla)$ Results obtained from Dambrine's method.

Fig. 15. - Minimum noise factor versus drain current. $f=8 \mathrm{GHz}(\bullet)$ Experimental results, $(\nabla)$ Results of the calculation from equation (9), ( $\mathbf{\nabla})$ Results obtained from Dambrine's method.

In Figures 10 to 15 we give the noise results. We have plotted the experimental ones, the calculated ones using équations (9) to (12) and the results obtained using the Dambrine's method [7].

In this method the authors use two assumptions:

- The first one is that the noise resistance $R_{\mathrm{n}}$ is (in the case of on wafer measurement) frequency independent. This fact is exact if we consider the intrinsic device, but we will show that it is not true when we take into account the gate, the source and the drain inductances even if they are very low.

- The second one concerns the correlation coefficient between the gate and the drain which is considered to be purely imaginary.

From this two considerations, it is then possible to obtain the noise parameters from a simple $50 \Omega$ noise measurement knowing the small-signal equivalent signal.

In Figure 10, we give the noise factor measurement (black squares) performed under $50 \Omega$ conditions versus the square of the frequency. We can see that the variation is linear and by means of the Dambrine's method we determine noise parameters which will be used further on. The white triangles are the results calculated from our noise parameters by means of the classical formula:

$$
F=F_{\min }+\frac{R_{n}}{G_{s}} \cdot\left[\left(G_{\mathrm{s}}-G_{\mathrm{opt}}\right)^{2}+\left(B_{\mathrm{s}}-B_{\mathrm{opt}}\right)^{2}\right] \text { with } B_{\mathrm{s}}=0 \text { and } G_{\mathrm{s}}=20 \mathrm{mS}
$$

The agreement is quite correct.

In Figure 11 we have plotted the minimum noise factor versus the frequency. The agreement is better than $0.05 \mathrm{~dB}$ over the whole frequency range. 
Figure 12 represents the phase of the optimal reflection coefficient versus the frequency, Dambrine's values are lightly lower than our results.

In Figure 13 where we have plotted the magnitude of the optimal reflection coefficient the disagreement is much more important and Dambrine's values are between 5 and $10 \%$ lower. Concerning the noise resistance (Fig. 14) Dambrine's value is constant of course and it seems that our results give a light dependence on the frequency. The calculated $R_{\mathrm{n}}$ decreases when frequency increases but the experimental parameter which is the most difficult to obtain with a good accuracy seems to increase. Nevertheless the difference between the three curves is lower than $2 \%$.

Then in Figure 15 we have plotted the minimum noise factor versus the drain current and the agreement is always very good. The minimum of the noise factor is obtained for a drain current of $9 \mathrm{~mA}$. For a higher current the noise factor increases relatively slowly. However when the drain current tends to zero the noise factor increases dramatically.

\section{Conclusions}

This work shows that it is possible with a good accuracy to predict, only by means of, the equivalent circuit determination, the noise behaviour of a PHEMT, assuming that the noise is only due to thermal noise.This fact suggests that the contribution of the hot carriers to the noise of the device may be neglected as predicted by Van der Ziel [14]. In this study we show that the Dambrine's method gives correct results excepted for the determination of the optimal reflection coefficient where the disagreement is about $6 \%$. This fact may be due to the initial hypothesis which neglects the extrinsic elements. Nevertheless this method needs an additional noise measurement to provide noise parameters. The experimental results given in this paper are in agreement with those found in literature $[19,20]$.

\section{Acknowledgments}

This work was supported by ELEN (European Laboratory on Electronic Noise).

\section{Appendix A}

$$
\begin{aligned}
& \alpha=g_{\mathrm{m}} R_{\mathrm{ds}} \sin (\omega \tau)+\omega\left\{R_{\mathrm{s}}\left(C_{\mathrm{gs}}+C_{\mathrm{gd}}\right)+R_{\mathrm{ds}} C_{\mathrm{gd}}+g_{\mathrm{m}} R_{d s} \cos (\omega \tau)\left[C_{\mathrm{gd}}\left(R_{\mathrm{s}}-R_{\mathrm{gd}}\right)\right.\right. \\
& \left.\left.-R_{\mathrm{ds}} C_{\mathrm{ds}}\right]\right\}+\omega^{2} g_{\mathrm{m}} R_{\mathrm{ds}} L_{\mathrm{s}} C_{\mathrm{gd}} \sin (\omega \tau) \\
& \beta=g_{\mathrm{m}} R_{\mathrm{ds}} \cos (\omega \tau)-\omega g_{\mathrm{m}} R_{\mathrm{ds}} \sin (\omega \tau)\left[C_{\mathrm{gd}}\left(R_{\mathrm{s}}-R_{\mathrm{gd}}\right)-R_{\mathrm{ds}} C_{\mathrm{ds}}\right]+\omega^{2} L_{\mathrm{s}}\left\{C_{\mathrm{gs}}+C_{\mathrm{gd}}\right. \\
& \left.+g_{\mathrm{m}} R_{\mathrm{ds}} C_{\mathrm{gd}} \cos (\omega \tau)\right\} \\
& a_{1}=R_{\mathrm{ds}}\left(1+\frac{C_{\mathrm{gd}}}{C_{\mathrm{gs}}}\right) \\
& a_{2}=\omega R_{\mathrm{ds}}\left\{C_{\mathrm{gd}}\left(R_{\mathrm{i}}+R_{\mathrm{gd}}\right)+R_{\mathrm{ds}} C_{\mathrm{ds}}\left(1+\frac{C_{\mathrm{gd}}}{C_{\mathrm{gs}}}\right)\right\} \\
& b_{1}=-\left(1+\frac{C_{\mathrm{gd}}}{C_{\mathrm{gs}}}\right) \\
& b_{2}=-\omega\left\{C_{\mathrm{gd}}\left(R_{\mathrm{i}}+R_{\mathrm{gd}}\right)+R_{\mathrm{ds}} C_{\mathrm{ds}}\left(1+\frac{C_{\mathrm{gd}}}{C_{\mathrm{gs}}}\right)\right\}
\end{aligned}
$$




$$
\begin{aligned}
& c_{1}=\left\{1+\frac{C_{\mathrm{gd}}}{C_{\mathrm{gs}}}\left[1+g_{\mathrm{m}} R_{\mathrm{ds}} \cos (\omega \tau)\right]\right\}-\omega g_{\mathrm{m}} R_{\mathrm{ds}}^{2} C_{\mathrm{ds}} \frac{C_{\mathrm{gd}}}{C_{\mathrm{gs}}} \sin (\omega \tau) \\
& c_{2}=g_{\mathrm{m}} R_{\mathrm{ds}} \frac{C_{\mathrm{gd}}}{C_{\mathrm{gs}}} \sin (\omega \tau)-\omega\left\{C_{\mathrm{gd}}\left(R_{\mathrm{i}}+R_{\mathrm{gd}}+R_{\mathrm{ds}}\right)+R_{\mathrm{ds}} C_{\mathrm{ds}}\left[2+2 \frac{C_{\mathrm{gd}}}{C_{\mathrm{gs}}}+g_{\mathrm{m}} R_{\mathrm{ds}} \frac{C_{\mathrm{gd}}}{C_{\mathrm{gs}}} \cos (\omega \tau)\right]\right\} \\
& d_{1}=c_{1} \\
& d_{2}=c_{2} \\
& e_{1}=-g_{\mathrm{m}} R_{\mathrm{ds}} \frac{C_{\mathrm{gd}}}{C_{\mathrm{gs}}} \cos (\omega \tau)-\omega g_{\mathrm{m}} R_{\mathrm{ds}}^{2} C_{\mathrm{ds}} \frac{C_{\mathrm{gd}}}{C_{\mathrm{gs}}} \sin (\omega \tau) \\
& e_{2}=g_{\mathrm{m}} R_{\mathrm{ds}} \frac{C_{\mathrm{gd}}}{C_{\mathrm{gs}}} \sin (\omega \tau)-\omega R_{\mathrm{ds}}\left\{C_{\mathrm{gd}}+g_{\mathrm{m}} R_{\mathrm{ds}} C_{\mathrm{ds}} \frac{C_{\mathrm{gd}}}{C_{\mathrm{gs}}} \cos (\omega \tau)\right\} \\
& f_{1}=-e_{1} \\
& f_{2}=-e_{2} \\
& g_{1}=-g_{\mathrm{m}} R_{\mathrm{ds}} \sin (\omega \tau)-\omega\left\{C_{\mathrm{gd}}\left(R_{\mathrm{ds}}+R_{\mathrm{s}}\right)+C_{\mathrm{gs}} R_{\mathrm{s}}+g_{\mathrm{m}} R_{\mathrm{ds}} \cos (\omega \tau)\left[C_{\mathrm{gd}}\left(R_{\mathrm{s}}-R_{\mathrm{gd}}\right)-C_{\mathrm{ds}} R_{\mathrm{ds}}\right]\right\} \\
& -\omega^{2} g_{\mathrm{m}} R_{\mathrm{ds}} C_{\mathrm{gd}} L_{\mathrm{s}} \sin (\omega \tau) \\
& g_{2}=-g_{\mathrm{m}} R_{\mathrm{ds}} \cos (\omega \tau)+\omega\left\{g_{\mathrm{m}} R_{\mathrm{ds}} \sin (\omega \tau) \cdot\left(R_{\mathrm{s}} C_{\mathrm{gd}}-R_{\mathrm{ds}} C_{\mathrm{ds}}-R_{\mathrm{gd}} C_{\mathrm{gd}}\right)\right\} \\
& -\omega^{2} L_{\mathrm{s}}\left\{C_{\mathrm{gs}}+C_{\mathrm{gd}}\left(1+g_{\mathrm{m}} R_{\mathrm{ds}} \cos (\omega \tau)\right)\right\} \\
& h_{1}=-g_{\mathrm{m}} R_{\mathrm{ds}} \sin (\omega \tau)+\omega\left\{C_{\mathrm{gs}}\left(R_{\mathrm{g}}+R_{\mathrm{i}}\right)+C_{\mathrm{gd}}\left(R_{\mathrm{g}}+R_{\mathrm{gd}}\right)+2 C_{\mathrm{ds}} R_{\mathrm{ds}}+g_{m} R_{\mathrm{ds}} \cos (\omega \tau)\right. \\
& \left.\times\left[C_{\mathrm{ds}} R_{\mathrm{ds}}+C_{\mathrm{gd}}\left(R_{\mathrm{ds}}+R_{\mathrm{gd}}\right)\right]\right\}+\omega^{2} g_{\mathrm{m}} R_{\mathrm{ds}} L_{\mathrm{g}} C_{\mathrm{gd}} \sin (\omega \tau) \\
& h_{2}=-\left\{1+g_{\mathrm{m}} R_{\mathrm{ds}} \cos (\omega \tau)\right\}-\omega g_{\mathrm{m}} R_{\mathrm{ds}} \sin (\omega \tau)\left(R_{\mathrm{g}} C_{\mathrm{gd}}+R_{\mathrm{ds}} C_{\mathrm{ds}}+R_{\mathrm{gd}} C_{\mathrm{gd}}\right) \\
& -\omega^{2}\left\{L_{\mathrm{g}}\left(C_{\mathrm{gs}}+C_{\mathrm{gd}}\right)+g_{\mathrm{m}} R_{\mathrm{ds}} C_{\mathrm{gd}} \cos (\omega \tau)\right\} \\
& i_{1}=-g_{\mathrm{m}} R_{\mathrm{ds}} \sin (\omega \tau)-\omega\left\{R_{\mathrm{s}} C_{\mathrm{gs}}+g_{\mathrm{m}} R_{\mathrm{ds}} \cos (\omega \tau)\left[R_{\mathrm{s}} C_{\mathrm{gd}}-R_{\mathrm{ds}} C_{\mathrm{ds}}-R_{\mathrm{gd}} C_{\mathrm{gd}}-\left(R_{\mathrm{g}}+R_{\mathrm{s}}\right) C_{\mathrm{gs}}\right]\right\} \\
& -\omega^{2}\left\{g_{\mathrm{m}} R_{\mathrm{ds}} \sin (\omega \tau)\left[L_{\mathrm{s}} C_{\mathrm{gd}}-\left(L_{\mathrm{g}}+L_{\mathrm{s}}\right) C_{\mathrm{gs}}\right]\right\} \\
& i_{2}=-g_{\mathrm{m}} R_{\mathrm{ds}} \cos (\omega \tau)+\omega\left\{g_{\mathrm{m}} R_{\mathrm{ds}} \sin (\omega \tau)\left[C_{\mathrm{gd}}\left(R_{\mathrm{s}}-R_{\mathrm{gd}}\right)-R_{\mathrm{ds}} C_{\mathrm{ds}}-\left(R_{\mathrm{g}}+R_{\mathrm{s}}\right) C_{\mathrm{gs}}\right]\right\} \\
& -\omega^{2}\left\{L_{\mathrm{s}}\left(C_{\mathrm{gs}}+C_{\mathrm{gd}}\right)+g_{\mathrm{m}} R_{\mathrm{ds}} \cos (\omega \tau)\left[L_{\mathrm{s}} C_{\mathrm{gd}}-\left(L_{\mathrm{s}}+L_{\mathrm{g}}\right) C_{\mathrm{gs}}\right]\right\} \\
& j_{1}=\omega\left\{\left(R_{\mathrm{g}}+R_{\mathrm{s}}+R_{\mathrm{i}}\right) C_{\mathrm{gs}}+\left(R_{\mathrm{gd}}+R_{\mathrm{g}}\right) C_{\mathrm{gd}}+R_{\mathrm{ds}} C_{\mathrm{ds}}+g_{\mathrm{m}} R_{\mathrm{ds}} \cos (\omega \tau)\left(R_{\mathrm{g}}+R_{\mathrm{s}}\right)\left(C_{\mathrm{gd}}-C_{\mathrm{gs}}\right)\right\} \\
& +\omega^{2} g_{\mathrm{m}} R_{\mathrm{ds}} \sin (\omega \tau)\left(L_{\mathrm{g}}+L_{\mathrm{s}}\right)\left(C_{\mathrm{gd}}-C_{\mathrm{gs}}\right) \\
& j_{2}=-1-\omega g_{\mathrm{m}} R_{\mathrm{ds}} \sin (\omega \tau)\left(R_{\mathrm{g}}+R_{\mathrm{s}}\right)\left(C_{\mathrm{gd}}-C_{\mathrm{gs}}\right) \\
& +\omega^{2}\left\{C_{\mathrm{gs}}\left(L_{\mathrm{g}}+L_{\mathrm{s}}\right)+L_{\mathrm{g}} C_{\mathrm{gd}}+\left(L_{\mathrm{g}}+L_{\mathrm{s}}\right) g_{\mathrm{m}} R_{\mathrm{ds}} \cos (\omega \tau)\left(C_{\mathrm{gd}}-C_{\mathrm{gs}}\right)\right\} \\
& k_{1}=-\omega\left\{C_{\mathrm{gd}}\left(R_{\mathrm{ds}}+R_{\mathrm{s}}\right)-g_{\mathrm{m}} R_{\mathrm{ds}}\left(R_{\mathrm{g}}+R_{\mathrm{s}}\right) C_{\mathrm{gs}} \cos (\omega \tau)\right\}+\omega^{2}\left(L_{\mathrm{g}}+L_{\mathrm{s}}\right) g_{\mathrm{m}} R_{\mathrm{ds}} C_{\mathrm{gs}} \sin (\omega \tau) \\
& k_{2}=-\omega g_{\mathrm{m}} R_{\mathrm{ds}}\left(R_{\mathrm{g}}+R_{\mathrm{s}}\right) C_{\mathrm{gs}} \sin (\omega \tau)-\omega^{2}\left\{L_{\mathrm{s}} C_{\mathrm{gd}}-\left(L_{\mathrm{g}}+L_{\mathrm{s}}\right) g_{\mathrm{m}} R_{\mathrm{ds}} C_{\mathrm{gs}} \cos (\omega \tau)\right\} \\
& l_{1}=\omega\left\{C_{\mathrm{gs}}\left(R_{\mathrm{g}}+R_{\mathrm{s}}+R_{\mathrm{i}}\right)+C_{\mathrm{gd}}\left(R_{\mathrm{g}}+R_{\mathrm{s}}+R_{\mathrm{ds}}+R_{\mathrm{gd}}\right)+2 C_{\mathrm{ds}} R_{\mathrm{ds}}\right. \\
& \left.+\left(R_{\mathrm{g}}+R_{\mathrm{s}}\right) g_{\mathrm{m}} R_{\mathrm{gd}} C_{\mathrm{gd}} \cos (\omega \tau)\right\}+\omega^{2}\left(L_{\mathrm{g}}+L_{\mathrm{s}}\right) g_{\mathrm{m}} R_{\mathrm{ds}} C_{\mathrm{gd}} \sin (\omega \tau)
\end{aligned}
$$




$$
\begin{aligned}
& \begin{array}{l}
l_{2}=-1-\omega\left(R_{\mathrm{g}}+R_{\mathrm{s}}\right) g_{\mathrm{m}} R_{\mathrm{ds}} C_{\mathrm{gd}} \sin (\omega \tau)+\omega^{2}\left\{\left(L_{\mathrm{g}}+L_{\mathrm{s}}\right) C_{\mathrm{gs}}+\left(L_{\mathrm{g}}+L_{\mathrm{s}}\right) g_{\mathrm{m}} R_{\mathrm{ds}} C_{\mathrm{gd}} \cos (\omega \tau)\right\} \\
m_{1}=-\omega R_{\mathrm{ds}}\left\{C_{\mathrm{gs}}\left(R_{\mathrm{g}}+R_{\mathrm{s}}+R_{\mathrm{j}}\right)+C_{\mathrm{gd}}\left(R_{\mathrm{g}}+R_{\mathrm{gd}}\right)+C_{\mathrm{ds}} R_{\mathrm{ds}}\right\} \\
m_{2}=R_{\mathrm{ds}}-\omega^{2} R_{\mathrm{ds}}\left\{C_{\mathrm{gs}}\left(L_{\mathrm{g}}+L_{\mathrm{s}}\right)+C_{\mathrm{gd}} L_{\mathrm{g}}\right\}
\end{array} \\
& \begin{array}{l}
Q=0 \\
\quad
\end{array} \\
& \quad+R_{\mathrm{d}}\left(d_{1} l_{2}-d_{2} l_{1}\right)+R_{\mathrm{i}}\left(e_{1} i_{2}-e_{2} i_{1}\right)+R_{\mathrm{gd}}\left(f_{1} k_{2}-f_{2} k_{1}\right) \\
& \quad \begin{array}{l}
g_{\mathrm{m}}\left(a_{1} m_{2}-a_{2} m_{1}\right)+R_{\mathrm{ds}}\left(b_{1} j_{2}-b_{2} j_{1}\right)+R_{\mathrm{s}}\left(c_{1} h_{2}-c_{2} h_{1}\right) \\
\quad
\end{array} \\
& \quad R_{\mathrm{g}}\left(g_{1}^{2}+g_{2}^{2}\right)+R_{\mathrm{s}}\left(h_{1}^{2}+h_{2}^{2}\right)+R_{\mathrm{i}}\left(i_{1}^{2}+i_{2}^{2}\right)+R_{\mathrm{ds}}\left(j_{1}^{2}+j_{2}^{2}\right)+R_{\mathrm{d}}\left(l_{1}^{2}+l_{2}^{2}\right)+g_{\mathrm{m}}\left(m_{1}^{2}+m_{2}^{2}\right)
\end{aligned}
$$

\section{References}

[1] Pospieszalski M. W., Modeling of noise parameters of MESFET's and MODFET's and their frequency and temperature dependence, IEEE Micr. T. 39 (1991) 724-731.

[2] Fukui $\mathrm{H}$., The noise performances of microwave transistor., IEEE trans. Electron Devices ED-13 (1966) 329-341.

[3] Fukui H., Design of microwave GaAs MESFET's for broad-band low-noise amplifiers., IEEE trans. MTT 27 (1979) 643-650.

[4] Gupta M. S. et al., Microwave noise characterization of GaAs MESFET's: determination of extrinsic noise parameters, IEEE Mıcr. T. 36 (1988) 745-751.

[5] Pucel R. A.et al., Signal and noise properties of gallium rsenide field effect transistors, Advan. Electron. and Electron Phys. 38 (1974) 195-265.

[6] Podell A. F., A fonctional GaAs FET noise model, IEEE trans. Electron Devnces ED-28 (1981) 511-517.

[7] Dambrine G. et al., A new method for on wafer noise measurement IEEE Micr. T. 41 (1993) 375-381.

[8] Cappy A., Propriétés physiques et performances potentielles des composants submicroniques à effet de champ: Structure conventionnelle et à gaz d'électrons bidimensionnel, Thèse de doctorat (Lille, 1986).

[9] Dambrine G. et al., A new method for determining the FET small-signal equivalent circuit., IEEE Mrcr. T. 36 (1988) 1151-1159.

[10] Diamant F. et al., "Measurement of the extrinsic series elements of a microwave MESFET under zero current condition", Proc. 12th European Microwave Conference (1982) pp. 451-456.

[11] Berroth M. et al., High-frequency equivalent circuit of AsGa FET's for large signal applications, IEEE Micr. T. 39 (1991) 224-229.

[12] Tasker P.J., Importance of source and drain resistance to the maximum fT of millimeter-wave MODFET's, IEEE Device Lett. 10 (1989) 291-293.

[13] Van Der Ziel A., Thermal noise in field-effect transistors, Proc. IRE 50 (1962) 1808-1812.

[14] Van Der Ziel A., Gate noise in field-effect transistors at moderately high frequencies, Proc. IRE 51 (1963) 461-467. 
[15] Wu E. N, and Van Der Ziel A., Induced-gate thermal noise in high electron mobility transistors, Solzd State Electronics 26 (1983) 639-642.

[16] Lane R. Q., The determination of device noise and parameters, Proc. IEEE 57 (1969) 1451-1462.

[17] Sannino M., On the determination of device noise and gain parameters, Proc. IEEE 68 (1980) 1343-1345.

[18] Application Note 1-90, Focus Microwaves.

[19] Wenger J., Quater-micrometer low-noise pseudomorphic GaAs HEMT's with extremely low dependence of the noise figure on drain-source current, IEEE Electron Device Lett. 14 (1993) 16-18.

[20] Henderson T. et al., Microwave performances of a quater-micrometer gate low-noise pseudomorphic InGaAs/AlGaAs modulation-doped field effect transistor, IEEE Electron Devnce Lett. 14 (1993) 16-18.

[21] Hillbrand H. et al., An efficient method for computer aided noise analysis of linear amplifier networks, IEEE Trans crrcuits syst. CAS23 4 (1976) 235-238. 